\title{
Las cintas suburetrales reducen la incontinencia urinaria post-cinugía reparadora de prolapso vaginal pero se asocian a mayores eventos adversos
}

Suburethral tapes reduce urinary incontinence after vaginal prolapse repair, but were associated with increased adverse events

Wei J y col. N Engl J Med 2012;366:2358-67.

\section{Objetivos}

Analizar la eficacia y seguridad de las cintas suburetrales para la profilaxis de la incontinencia de orina $(\mathrm{IO})$ postquirúrgica en mujeres sometidas a cirugía reparadora de prolapso vaginal.

\section{Diseño, lugar y pacientes}

Estudio multicéntrico, aleatorizado, doble ciego y controlado, realizado en siete centros en EE.UU. Participaron 337 mujeres candidatas a cirugía reparadora de prolapso vaginal, con síntomas o sensación de bulto vaginal sin IO de esfuerzo.

\section{Intervención}

Las pacientes fueron aleatorizadas por bloques a recibir cinta suburetral (Gynecare-TVT; $n=165$ ) o a dos falsas incisiones suprapúbicas superficiales de $1 \mathrm{~cm}$, que simulaban las inci- siones de las cintas $(n=172)$. Se estratificaron por cirujano y por tipo de cirugía de prolapso.

\section{Medición de resultados principales}

Fueron evaluados dos resultados primarios: 1) prevalencia de IO (de esfuerzo, de urgencia o mixta), 2) necesidad de tratamiento de la 10 a los 3 y 12 meses. Como resultados secundarios fueron evaluados puntajes de incontinencia y calidad de vida, eventos adversos serios, complicaciones atribuibles a las cintas y eventos adversos inesperados. El análisis fue por intención de tratar.

\section{Resultados}

A los 3 y 12 meses, la tasa de IO fue menor en el grupo "cinta". Ver tabla 1.

Tabla 1. Eficacia a los 3 y 12 meses del tratamiento de la incontinencia urinaria con cintas suburetrales.

\begin{tabular}{|c|c|c|c|c|}
\hline & & Cintas sub-uretrales ( $n=165)$ & Control ( $n=172)$ & Diferencia (IC95\%) \\
\hline \multirow[t]{2}{*}{ Incontinencia urinaria } & $3 \mathrm{~m}$ & $39(23,6 \%)$ & $85(49,4 \%)$ & $-25,8 \%(-36,1$ a 15,5$)$ \\
\hline & $12 \mathrm{~m}$ & $45(27,3 \%)$ & $74(43 \%)$ & $-15,7 \%(-26 a-5,5)$ \\
\hline \multirow[t]{2}{*}{ Prueba de esfuerzo de la tos positiva } & $3 \mathrm{~m}$ & 10/158 (6,5\%) & $54 / 157(34,4 \%)$ & $-28,1 \%(-37,0$ a $-19,2)$ \\
\hline & $12 \mathrm{~m}$ & $5 / 143(3,5 \%)$ & $31 / 151(20,5 \%)$ & $-17,0 \%(-24,5 a-9,5)$ \\
\hline \multirow[t]{2}{*}{ Sintomas de incontinencia } & $3 \mathrm{~m}$ & $15 / 160(9,4 \%)$ & $41 / 165(24,8 \%)$ & $-15,5 \%(-23,7$ a $-7,3)$ \\
\hline & $12 \mathrm{~m}$ & $18 / 158(11,4 \%)$ & $30 / 160(18,8 \%)$ & $-7,4 \%(-15,2$ a 0,5$)$ \\
\hline \multicolumn{2}{|l|}{ Necesidad de otro tratamiento ${ }^{a}$} & $11 / 164(6,7 \%)$ & $13 / 172(7,6 \%)$ & $-0,9 \%(-6,45$ a 4,7$)$ \\
\hline
\end{tabular}

a Ajustado por cirujano y por tipo de cirugía.

Fue necesario tratar con cinta uretral a seis pacientes para evitar un episodio de incontinencia a los 12 meses. La tasa de perforación de la vejiga fue mayor en el grupo intervenido $(6,7$ vs $0 \%$, así como la de infección del tracto urinario (31\% vs $18,3 \%)$, las complicaciones hemorrágicas mayores $(3,1 \%$ vs $0 \%)$, y el vaciado vesical incompletos a las seis semanas de la cirugía $(3,7 \%$ frente a $0 \%$ ) ( $p \leq 0,05$ para todas las comparaciones).

\section{Conclusiones}

La colocación de una cinta suburetral a modo profiláctico durante la cirugía de prolapso vaginal resultó en una menor tasa de incontinencia urinaria a los 3 y 12 meses, pero presentó mayores tasas de eventos adversos.

Fuentes de financiamiento: Eunice Kennedy Shriver National Institute of Child Health and Human Development and NIH Office of Research on Women's Health. Conflictos de intereses: no referidos.

\section{Comentario}

La IO afecta entre $4 \%$ y $40 \%$ de las mujeres, predominando en las mayores de 50 años ${ }^{1,2}$. Clásicamente, la retrosuspensión colpopúbica abdominal abierta ha sido considerada como el tratamiento de referencia, con una efectividad del $69 \%$ a $90 \%{ }^{3}$. Sin embargo, sus complicaciones severas, su morbilidad y su alta tasa de recidiva han conducido a que se investiguen nuevas tecnologías ${ }^{4}$, como la exitosa introducción en 1996 de los "sling" (cinta libre de tensión o TVT, del inglés Tension Free Vaginal Tape). Con el objetivo de reducir la incidencia de hematomas extensos, y de lesiones del intestino y de los vasos sanguíneos, se desarrolló en 2001 un nuevo dispositivo denominado cinta libre de tensión transobturador (TVT-O), que se convirtió en el actual estándar de cuidado para esta condición $^{5-9}$; que a pesar de la escasez de datos respecto de su seguridad y eficacia fue avalado para la reparación del prolapso pélvico por un Comité de la Administración de Drogas y Medicamentos de EE.UU. (FDA).

\section{Conclusiones del comentador}

La decisión de realizar cirugía profiláctica anti-incontinencia debe tener en cuenta los objetivos y los deseos de la paciente, la habilidad y experiencia del cirujano y los riesgos y beneficios potenciales para cada paciente en particular.

Cintia Spira [ Instituto de Efectividad Clínica y Sanitaria, cspira@iecs.org.ar ]

Spira C. Las cintas suburetrales reducen la incontinencia urinaria post-cirugía reparadora de prolapso vaginal pero se asocian a mayores eventos adversos. Evid Act Pract Ambul. Abr-Jun 2013;16(2):48. Comentado de: Wei J y col. A Midurethral Sling to Reduce Incontinence after Vaginal Prolapse Repair. N Engl J Med. 2012; 366(25):2358-2367.

\section{Referencias}

1. Luber K. The definition, prevalence, and risk factors for stress urinary incontinence. Rev Urol. 2004:6(3:S3).

2. Tejerizo J. Incontinencia de orina femenina. Sling: diferentes tipos. Comité de Educación Médica Continua Sociedad Argentina de Urología. 2003.

3. Liapis A y col. Tension-Free Vagina Tape versus Tension- Free Vaginal Tape Obturador in Women with Stress Urinary Incontinence. Gynecol Obstet Invest. 2006;62(3):160-4.

4. Jelovsek E y col. Stress urinary incontinence in women: Choosing a primary surgical procedure. www.uptodate.com. 2012.

5. Ogah J y col. Minimally invasive synthetic suburethral sling operations for stress urinary incontinence in women. Cochrane Database Syst Rev 2009;4:CD006375.

6. Ward K y col. Tension-free vaginal tape versus colposuspension for primary urodynamic stress incontinence: 5-year follow up. BJOG 2008;115:226-33.

7. Labrie J y col. Protocol for Physiotherapy Or TVT Randomised Efficacy Trial (PORTRET): a multicentre randomised controlled trial to assess the cost-effectiveness of the tension free vaginal tape versus pelvic floor muscle training in women with symptomatic moderate to severe stress uninary incontinence. BMC Womens Health. 2009;9:24.

8. Araco F y col. TVT-O vs TVT: a randomized trial in patients with different degrees of urinary stress incontinence. Int Urogynecol J Pelvic Floor Dysfunct. 2008 Jul;19(7):917-26.

9. Funk J y col. Trends in the surgical management of stress urinary incontinence. Obstet Gynecol. 2012;119(4):845-51.

10. Urogynecologic Surgical Mesh: Update on the Safety and Effectiveness of Transvaginal Placement for Pelvic Organ Prolapse. 2011. U.S. Food and Drug Administration 